\section{Análise epidemiológica das \\ causas externas em unidades \\ de urgência e emergência em \\ Cuiabá/Mato Grosso}

\section{Epidemiological analysis of external}

causes at urgency and emergency

services in Cuiabá/Mato Grosso

\section{Ligia Regina de Oliveira ${ }^{1}$}

\section{Maria Helena Prado de Mello Jorge ${ }^{2}$}

${ }^{1}$ Departamento de Saúde Coletiva, Instituto de Saúde Coletiva - Universidade Federal de Mato Grosso.

${ }^{2}$ Departamento de Epidemiologia, Faculdade de Saúde Pública - USP.

Artigo baseado na tese Subsídios para implantação de um sistema de vigilância de causas externas no município de Cuiabá/MT apresentada na Faculdade de Saúde Pública da Universidade de São Paulo em fevereiro de 2007.

Instituição onde o trabalho foi desenvolvido: Secretaria Municipal de Saúde de Cuiabá/Mato Grosso

Auxílio financiamento: Programa de Qualificação Institucional/Coordenação de Aperfeiçoamento de Pessoal de Nível Superior - PQI/CAPES

Agradecimentos: À Secretaria Municipal de Saúde de Cuiabá/MT pelo apoio logístico e disponibilização de profissionais para coleta dos dados.

Correspondência: Ligia Regina de Oliveira. Rua E, n॰163 - Casa 12 - Residencial dos Ipês - Village

Flamboyant - CEP 78035-400 Cuiabá/MT. E-mail:Irdoliveira@terra.com.br

\section{Resumo}

Objetivo: Apesar da importância das causas externas como problema de saúde pública, pouco se conhece sobre a demanda de serviços de urgência e emergência. Este estudo tem como objetivo caracterizar a morbidade por causas externas em unidades de urgência e emergência do município de Cuiabá/ MT. Método: Trata-se de um estudo transversal e descritivo. Foram analisadas 3.786 vítimas de causas externas atendidas pelas cinco unidades de urgência e emergência da Secretaria Municipal de Saúde de Cuiabá/ MT, no período de 1 de maio a 30 de junho de 2005. Resultados: Aproximadamente $88 \%$ dos atendimentos se referiam a vítimas de acidentes, $9 \%$ corresponderam a agressões e $2 \%$ a lesões autoprovocadas. Os acidentes de transportes representaram $22 \%$ dos atendimentos, sendo os motociclistas as principais vítimas (49\%); as quedas foram as causas mais freqüentes no grupo de outras causas externas de traumatismos acidentais. A prevalência em homens superou a de mulheres. A maior parte das vítimas era menor de 40 anos (79\%). No entanto, a análise por tipo de causa externa apresenta resultados diferentes segundo sexo e faixa etária. Cerca da metade dos eventos ocorreu em casa e, em sua maioria, as vítimas receberam alta após o atendimento, sendo que a taxa de mortalidade foi baixa $(0,4 \%)$. Conclusão: Os resultados revelam a importância da análise sistemática dos dados referentes às vítimas de acidentes e violência atendidas em unidades de urgência e emergência, como complemento às informações sobre mortalidade e morbidade hospitalar visando o monitoramento dessas causas.

Palavras-chave: Causas Externas. Acidentes. Violências. Morbidade. Emergências. Sistemas de Informação. 


\section{Abstract}

Objective: Despite the importance of external causes as a public health problem, little is known about the demand for emergency services. This study aims to characterize the morbidity due to external causes in emergency units of Cuiabá/MT. Method: This is a cross-sectional and descriptive study. We analyzed 3,786 victims of external causes seen at five emergency units of Cuiabá/MT Health Department in the period from May 1 to June 30, 2005. Results: Approximately $88 \%$ of cases involved victims of accidents, $9 \%$ were victims of assault, and $2 \%$ were victims of self-injury. Transportation accidents accounted for $22 \%$ of cases, whose main victims were motorcyclists $(49 \%)$; falls were the most frequent causes in the group of other external causes of accidental injuries. The prevalence in men surpassed that of women. Most of the victims were younger than 40 years (79\%). However, the analysis by type of external cause presents different results by gender and age. Nearly half of the events occurred at home, most of the victims were discharged after treatment, and the mortality rate was low $(0.4 \%)$. Conclusion: The results show the importance of the systematic analysis of data on victims of accidents and violence seen at emergency services, to add information on hospital mortality and morbidity aimed at the surveillance of these causes.

Keywords: External Causes. Accidents. Violence. Morbidity. Emergency. Information System.

\section{Introdução}

As causas externas - acidentes e violências - configuram -se como um dos mais importantes temas na atualidade, adquirindo caráter epidêmico e convertendo-se em um dos problemas mais sérios de Saúde Pública no mundo ${ }^{1,2}$. Em muitas áreas do Brasil, já representa a segunda causa de morte; mostrando uma tendência crescente $\mathrm{e}^{3-5}$.

Uma das abordagens da Saúde Pública em relação à violência, apresentada no Relatório Mundial sobre Violência e Saúde ${ }^{2}$, trata da necessidade de estratégias para revelação do máximo possível de conhecimento básico a respeito de todos os aspectos da violência - através da coleta sistemática de dados sobre sua magnitude, seu alcance, suas características e conseqüências. A $\mathrm{OMS}^{2}$ (2002) refere que, na maioria dos países do mundo, normalmente, não há dados sistemáticos sobre resultados não fatais. Mesmo quando há dados disponíveis, a qualidade das informações pode não ser adequada, uma vez que, em grande parte, as instituições mantêm registros voltados para seus próprios objetivos, podendo, assim, gerar dados incompletos ou informações que não levam à compreensão adequada sobre a violência. É comum verificar que os registros médicos podem conter informações de diagnóstico sobre a lesão e o tratamento, mas não as circunstâncias do acidente ou a violência relacionadas à lesão² .

No Brasil, o impacto da violência no setor saúde pode ser estudado por meio de várias fontes, nenhuma delas, entretanto, totalmente completa e correta ${ }^{5-7}$. Uma das limitações é a não inclusão dos muitos casos de acidentes e violências que recebem atendimento apenas nas emergências ou nos pronto-socorros, apesar de ser nesses serviços que essas causas adquirem maior visibilidade ${ }^{8-10}$. Entretanto, segundo alguns autores ${ }^{2,3}$, o quadro epidemiológico relativo a cada tipo de causa externa e de vítima precisa ser estabelecido e, para tanto, cada uma das fontes pode ser utilizada como complemento das demais, a fim de que a informação possa ser melhorada. 
A relevância dos acidentes e violências em Cuiabá, capital do Estado de Mato Grosso, é revelada pelo quadro de mortalidade por causas externas. No período de 1991 a 2000, entre as capitais brasileiras, Cuiabá apresentou um dos maiores crescimentos das taxas de mortalidade por acidentes de transporte ${ }^{11}$ e o maior incremento da taxa de mortalidade por armas de fogo $(690,0 \%)^{12,13}$. Em 2005, entre as capitais brasileiras, Cuiabá apresentou a sexta maior taxa de mortalidade por causas externas na população geral (90,9/100.000 hab), a quinta em acidentes de transporte e a sexta em homicídios ${ }^{14-16}$. Os dados de morbidade hospitalar para Cuiabá revelam-se acima dos índices do Brasil, da região Centro-Oeste e do Estado de Mato Grosso ${ }^{14}$.

Em 2005, visando a complementação dos dados de mortalidade e morbidade hospitalar existentes, e em conseqüência, qualificar o conhecimento sobre os acidentes e violências, foi realizado este estudo em unidades de urgência e emergência do Município de Cuiabá. Neste sentido, o presente estudo buscou verificar a magnitude desses agravos e caracterizar, segundo algumas variáveis de importância epidemiológica, as vítimas atendidas nessas unidades. Deve ser destacado que, na época do desenvolvimento dessa pesquisa, ainda não se dispunha de dados dessa natureza para o município estudado. Somente em setembro de 2006, o Ministério da Saúde iniciou, em alguns municípios, incluindo Cuiabá, o levantamento de dados em unidades sentinelas ${ }^{17}$.

\section{Método}

Trata-se de um estudo descritivo, de corte transversal. A população foi constituída por todas as vítimas de acidentes, lesões autoprovocadas e agressões, residentes ou não no município de Cuiabá/MT, atendidas nas seis unidades de urgência e emergência da Secretaria Municipal de Saúde (Pronto Socorro e cinco Policlínicas) que compõe a rede pública de urgência e emergência, cobrindo mais de $90 \%$ deste tipo de demanda. A coleta se deu no período de 1 de maio a 30 de junho de 2005; por se tratar de uma etapa de uma tese de doutorado, esses meses foram selecionados considerando-se o tempo disponível para o levantamento e a análise de dados, já que foi constatado que não há variabilidade sazonal para a mortalidade e morbidade hospitalar em Cuiabá.

Foram englobados todos os indivíduos que apresentaram conseqüências de causas externas, independente de sua gravidade, considerando-se os atendimentos de primeira consulta e descartando-se os retornos gerados por este primeiro atendimento. Contudo, se um mesmo paciente buscasse o serviço de saúde, tendo sido acometido por outro acidente ou violência, seria alvo de nova notificação, ou seja, considerado outro caso. Totalizaram 3.786 atendimentos por causas externas no período do estudo. Foram excluídas as pessoas que buscaram esses serviços por outros motivos.

A coleta de dados se deu através de um instrumento, denominado Ficha de Notificação de Causas Externas, criado para essa finalidade, que constava dos seguintes blocos e variáveis:

- Identificação da vítima: nome, data de nascimento, idade, nome da mãe, endereço, sexo, ocupação, estado civil, escolaridade, cor/raça, como chegou à unidade

- Circunstâncias em que ocorreu o evento:

- Acidente de Trabalho

- Acidente de transporte (tipo, qualidade da vítima, uso de proteção

- Demais acidentes

- Lesões autoprovocadas voluntariamente

- Agressão (tipo, qualidade do agressor)

- Intencionalidade ignorada

- Sob efeito de drogas (tipo)

- Suspeita de maus tratos

- Identificação da ocorrência do evento: Data, hora, local, endereço

- Identificação da lesão:

- Gravidade

- Natureza

- Parte do corpo atingida 
- Saída: Data, hora, tipo (alta, óbito, internado, transferência, a revelia)

A coleta dos dados foi efetuada por profissionais de saúde das próprias unidades, capacitados para tal.

Os acidentes e violências foram categorizados segundo a Classificação Internacional de Doenças - $10^{\text {a }}$ revisão ${ }^{18}$.

Utilizou-se do banco de dados MS-SQL SERVER 2000 e do programa Microsoft Office Excel 2003 para o armazenamento e o processamento dos dados. Os dados foram apresentados em freqüências absolutas e relativas.

Cabe destacar que alguns campos da Ficha de notificação de causas externas não foram preenchidos adequadamente, o que interferiu na análise dos dados. Por este motivo, são apresentados nas tabelas os registros obtidos para cada uma das variáveis.

O estudo foi aprovado pelo Comitê de Ética em Pesquisa da Faculdade de Saúde Pública da Universidade de São Paulo em 26 de março de 2004 (Protocolo de Pesquisa $\left.\mathrm{n}^{\circ} 1.066\right)$.

\section{Resultados}

Do total de registros, $97,3 \%$ referiamse a atendimentos realizados no Hospital e Pronto Socorro Municipal de Cuiabá. A maioria era residente em Cuiabá $(89,2 \%)$ e, em geral, as vítimas foram conduzidas ao atendimento com veículo próprio ou de conhecido (53,0\%). No entanto, o Serviço de Atendimento Médico de Urgência (SAMU) e o Sistema Integrado de Atendimento ao Trauma em Emergência (SIATE) tiveram papel importante já que foram o meio de locomoção utilizado por $42,1 \%$ das vítimas de acidentes de transporte, em $21,1 \%$ das lesões autoprovocadas e em $18,7 \%$ das agressões.

Os atendimentos se concentraram especialmente nos finais de semana $(45,1 \%)$; cerca de metade das agressões $(48,9 \%) \mathrm{e}$ mais de 1/3 dos acidentes de transporte (35,5\%) ocorreram nos sábados e domingos, enquanto as lesões autoprovocadas foram mais freqüentes aos domingos e às segundas-feiras $(45,6 \%)$.

Foi observado que a proporção de homens superou a de mulheres numa razão de sexos igual a 2. A análise da razão de sexo segundo o tipo de causa externa mostrou diferenças relevantes: enquanto para as vítimas de agressões, a razão foi de 3,5, para as vítimas de lesões autoprovocadas foi de 0,4 (Tabela 1).

Em relação à idade, a maioria das vítimas era menor de 40 anos $(79,2 \%)$,

Tabela 1 - Vítimas atendidas (\%) nas unidades de urgência e emergência segundo tipo de causa externa e sexo. Cuiabá/MT, maio a junho, 2005.

Table 1 - Victims (\%)seen at emergency services according to type of external cause and gender. Cuiabá/MT, May - June, 2005.

\begin{tabular}{lcccc}
\hline Tipo de causa externa & Total' $^{1}(\%)$ & \multicolumn{3}{c}{ Sexo } \\
\cline { 3 - 5 } & & Masculino (\%) & Feminino (\%) & Razão M/F \\
\hline Acidente de Transporte & 21,8 & 23,3 & 18,6 & $2,6: 1$ \\
Demais Acidentes $^{2}$ & 65,8 & 63,7 & 70,1 & $1,9: 1$ \\
Lesões Autoprovocadas & 1,7 & 0,7 & 3,7 & $0,4: 1$ \\
Agressões & 8,7 & 10,1 & 5,9 & $3,5: 1$ \\
Intenção Indeterminada & 2,0 & 2,1 & 1,8 & $2,5: 1$ \\
\hline Total $^{2}$ & $\mathbf{1 0 0 , 0}$ & $\mathbf{1 0 0 , 0}$ & $\mathbf{1 0 0 , 0}$ & $\mathbf{2 , 0 : 1}$ \\
\hline
\end{tabular}

'Excluindo três casos de "demais acidentes" onde o sexo foi ignorado

${ }^{2}$ Outras causas externas de traumatismos acidentais

"Excluded three cases of "other accidents" in which gender was unknown

${ }^{2}$ Other external causes of accidental trauma 
Tabela 2 - Vítimas atendidas (\%) nas unidades de urgência e emergência segundo tipo de causa externa e faixa etária. Cuiabá/MT, maio a junho, 2005.

Table 2 - Victims (\%) seen at emergency services according to type of external cause and age. Cuiabá/MT, May - June, 2005.

\begin{tabular}{lcccccc}
\hline Idade (em anos) & \multicolumn{7}{c}{ Tipo de Causa Externa } \\
\cline { 2 - 7 } & $\begin{array}{c}\text { Acidente } \\
\text { Transporte }\end{array}$ & $\begin{array}{c}\text { Demais } \\
\text { Acidentes }^{1}\end{array}$ & Lesão Autopro. & Agressão & $\begin{array}{c}\text { Intenção } \\
\text { Indeterm. }\end{array}$ & $\begin{array}{c}\text { Total } \\
\text { N }(\%)\end{array}$ \\
\hline Menor 1 & - & 0,4 & - & - & - & $11(0,3)$ \\
1 a 4 & 2,1 & 10,3 & - & 0,6 & 8,1 & $279(7,4)$ \\
5 a 9 & 6,8 & 12,6 & - & 2,7 & 13,5 & $388(10,3)$ \\
10 a 14 & 6,1 & 8,8 & 3,1 & 2,7 & 8,1 & $284(7,5)$ \\
15 a 19 & 11,0 & 9,1 & 10,9 & 15,5 & 9,5 & $381(10,1)$ \\
20 a 29 & 40,0 & 21,5 & 46,9 & 36,5 & 24,3 & $1.027(27,3)$ \\
30 a 39 & 18,3 & 14,5 & 18,8 & 24,9 & 13,5 & $613(16,3)$ \\
40 a 49 & 9,4 & 11,2 & 14,1 & 11,2 & 6,8 & $405(10,8)$ \\
50 a 59 & 3,9 & 6,1 & 3,1 & 3,6 & 8,1 & $203(5,4)$ \\
60 a 69 & 1,6 & 3,1 & - & 1,8 & 2,7 & $97(2,6)$ \\
70 a 79 & 0,7 & 1,5 & 3,1 & 0,3 & 4,1 & $49(1,3)$ \\
80 e mais & 0,2 & 0,9 & - & - & 1,4 & $26(0,7)$ \\
Sub-Total & $821(100,0)$ & $2.475(100,0)$ & $64(100,0)$ & $329(100,0)$ & $74(100,0)$ & $3.763(100,0)$ \\
Ignorado & 3 & 17 & - & 1 & 2 & 23 \\
\hline Total & $\mathbf{8 2 4}$ & $\mathbf{2 . 4 9 2}$ & $\mathbf{6 4}$ & $\mathbf{3 3 0}$ & $\mathbf{7 6}$ & $\mathbf{3 . 7 8 6}$ \\
\hline
\end{tabular}

'Outras causas externas de traumatismos acidentais / ' Other external causes of accidental trauma

*excluído idade ignorada/*excluded ignored age

chamando a atenção para o fato de que os menores de 10 anos representaram $18,0 \%$ de todos os atendimentos, sendo o segundo grupo mais freqüente (Tabela 2). Quando se observa o tipo de causa externa foram verificadas diferenças entre os diversos grupos etários: cerca de $50 \%$ das lesões autoprovocadas ocorreram no grupo etário de 20 a 29 anos; nesse mesmo grupo ocorreram 40,0\% dos acidentes de transporte $\mathrm{e}$ $36,5 \%$ das agressões, já os demais acidentes foram mais freqüentes em menores de 10 anos $(22,5 \%)$.

A análise quanto à escolaridade, estado civil e raça/cor, ficou prejudicada pelo alto índice de informações ignoradas: 29\%, 48\% e $31 \%$ respectivamente.

A importância dos acidentes nos atendimentos em unidades de urgência e emergência é observada pelos altos percentuais de acidentes de transporte e outras causas externas de lesões acidentais ${ }^{18}$, nomeadas neste estudo de 'demais acidentes', que fo- ram responsáveis por $87,6 \%$ dos atendimentos nas unidades estudadas. As agressões representaram $8,7 \%$ e as lesões autoprovocadas, $1,7 \%$ da demanda. Em apenas 2,0\% dos registros não foi identificado o tipo de causa externa (Tabela 1).

Destaca-se o fato de que acidente de trabalho foi referido por cerca de $21 \%$ das vítimas de acidentes, com 10 anos ou mais de idade.

Os motociclistas foram as principais vítimas de acidentes de transporte (49,1\%). Entre os demais acidentes, as quedas, especialmente as de outras alturas, foram as mais comuns; ainda no grupo de demais acidentes, picadas de animais e contato com corpo estranho tiveram papel importante na demanda dos serviços de urgência e emergência em Cuiabá (Tabela 3).

Quanto às violências observou-se que são diferentes os meios utilizados. A quase totalidade das lesões autoprovocadas foi decorrente de envenenamento $(93,4 \%)$, 
Tabela 3 - Vítimas de acidentes atendidas (\%) nas unidades de urgência e emergência segundo tipo de acidente. Cuiabá/MT, maio a junho, 2005.

Table 3 - Victims (\%) seen at emergency services according to type of accident. Cuiabá/MT, May June, 2005.

\begin{tabular}{lc}
\hline Tipo de acidente & $\%$ \\
\hline Acidente de transporte ( $n=824)$ & 21,1 \\
Ciclista & 4,0 \\
Condutor & 49,1 \\
Motociclista & 10,7 \\
Passageiro & 14,5 \\
Pedestre & 0,6 \\
Outros & \\
Demais Acidentes' ( $n=2.492)$ & 47,1 \\
Quedas & 68,9 \\
$\quad$ Outras alturas & 24,6 \\
$\quad$ Própria altura & 6,6 \\
$\quad$ Outro & 4,0 \\
Queimaduras & 3,1 \\
$\quad$ Choque elétrico & 21,6 \\
$\quad$ Fogo & 8,2 \\
$\quad$ Produtos químicos & 55,8 \\
Substância quente & 11,3 \\
Outros & 48,9 \\
Outros acidentes & 16,2 \\
Corpo estranho & 4,9 \\
Intoxicação & 8,0 \\
Objeto lançado & 24,3 \\
Picada & 46,6 \\
Outros & \\
\hline Outras causas externas de traumatismos acidentais \\
'Other external causes of accidental trauma & \\
\hline
\end{tabular}

enquanto os meios mais utilizados contra as vítimas de agressão foram a agressão física $(54,0 \%)$, o uso de instrumento cortante $(18,0 \%)$ e de arma de fogo $(16,2 \%)$ (Tabela 4).

Em relação às agressões deve ser destacado que mais da metade das vítimas $(55,2 \%)$ conhecia o agressor, fosse ele da família $(15,6 \%)$ ou não $(39,6 \%)$.

Grande parte $(44,1 \%)$ dos acidentes e violências ocorreu em residência. Este percentual variou de acordo com o tipo de causa externa: para lesões autoprovocadas o percentual de ocorrência em residência foi de 94,6\%, entre os demais acidentes foi de $58,5 \%$ e, entre as agressões, $29,3 \%$.

Nas situações onde foi possível observar o uso de drogas, 7,3\% das vítimas estavam sob efeito de álcool e $0,8 \%$ sob o efeito de outras drogas; no caso de agressões, $36,3 \%$ das vítimas estavam alcoolizadas e nos acidentes de transporte $8,1 \%$ estavam sob o efeito dessa droga. Os grupos etários que mais fizeram uso de álcool foram o de 20 a $29(39,1 \%)$ e o de 30 a 39 anos $(31,6 \%)$, esses também concentraram o maior uso de outras drogas, com $66,7 \%$ dos casos.

A taxa de mortalidade foi baixa $(0,4 \%)$, tendo sido a maior encontrada nas agressões $(2,5 \%)$ e a menor entre os demais acidentes $(0,1 \%)$. Noventa por cento das vítimas receberam alta após o atendimento nas unidades de urgência e emergência.

Tabela 4 - Vítimas de lesões autoprovocadas e agressões (\%) segundo meio utilizado. Cuiabá/ MT, maio a junho de 2005.

Table 4 - Victims of suicide attempts and assaults (\%) according to instrument. Cuiabá/MT, MayJune, 2005.

\begin{tabular}{lcc}
\hline Meio/instrumento & Lesões autoprovocadas ${ }^{1}(\%)$ & Agressões $^{2}(\%)$ \\
\hline Agressão física & - & 54,0 \\
Arma de fogo & 1,6 & 16,2 \\
Instrumento contundente & 1,6 & 8,8 \\
Instrumento cortante & 3,0 & 18,0 \\
Envenenamento & 93,8 & 1,2 \\
Outros & - & 1,8 \\
\hline
\end{tabular}

${ }^{1}$ Lesões autoprovocadas $=64 /{ }^{\prime}$ Autoprovoked injuries $=64$

${ }^{2}$ Agressões $=330 /{ }^{2}$ Assaults $=330$ 


\section{Discussão}

Uma importante limitação na análise desse estudo se deve ao processo de comparação dos dados referentes à demanda de serviços de urgência e emergência, seja devido à deficiência dos sistemas de informação ${ }^{2,3}$ ou pela escassez de publicações sobre o tema ${ }^{7,19}$. Grande parte das produções científicas nacionais referentes a causas externas está relacionada à mortalidade ou morbidade hospitalar ${ }^{7,19}$. Quanto à produção internacional, apenas $0,8 \%$ focaliza as causas externas em geral; em sua maioria o enfoque é sobre um tipo de causa especifica, grupo populacional ou relacionado à lesão; as fontes de dados desses estudos são, em geral, os prontuários médicos- hospitalares e declaração de óbito? .

Reconhece-se, ainda, que este estudo oferece somente uma perspectiva pontual da realidade sobre os acidentes e violências em Cuiabá, já que revela apenas os dados sobre vítimas não fatais atendidas em unidades de urgência e emergência por um curto período de tempo. No entanto, permitiu uma visualização das características desses eventos e de suas vítimas, que inexistia, para o local estudado e que podem contribuir substancialmente para o planejamento das ações de saúde.

A qualidade do preenchimento da Ficha de notificação de causas externas não foi homogênea para todas as variáveis. Portanto, esse é um fato a ser considerado ao analisar os resultados desse estudo, contudo é também de relevância no sentido que aponta para as possíveis limitações deste tipo de levantamento, qual seja o realizado em unidades de urgência e emergência.

A análise segundo o tipo de causa externa revelou que os acidentes fazem um maior número de vítimas que procuram as unidades de urgência e emergência em Cuiabá, sendo os demais acidentes os mais freqüentes $(65,8 \%)$. Alguns autores afirmam que há predominância deste tipo de causa externa também na morbidade hospita$\operatorname{lar}^{7,20,21}$. Mesquita Filho e Mello Jorge (2007) ${ }^{22}$ constataram que $73,1 \%$ dos atendimentos por causas externas em uma unidade de urgência e emergência de Pouso Alegre/ MG foram pelos demais acidentes. Por outro lado, os resultados deste estudo diferem de outros, como os encontrados em um hospital do município de São Paulo ${ }^{23}$, de um hospital de São José dos Campos/SP ${ }^{24}$ e em outro de Alta Floresta/MT ${ }^{25}$, nos quais os demais acidentes representaram, respectivamente, $39,0 \%, 35,3 \%$ e $54,8 \%$ da demanda.

Outro aspecto a ser considerado, quanto ao tipo de causa externa, são as diferenças entre os resultados aqui apresentados e o perfil de morbidade hospitalar e mortalidade para Cuiabá. O grupo de demais acidentes representou, em 2007, 75,7\% das internações por causas externas e apenas $10,0 \%$ das mortes por essas causas em $2005^{14}$.

Este grupo de causas externas apresenta-se com uma grande variedade de eventos. Neste estudo, assim como outros ${ }^{23-25}$, as quedas prevaleceram nos atendimentos de urgência e emergência. Contudo, diferem dos resultados de Mesquita e Mello Jorge $(2007)^{22}$ cujo percentual de atendimentos resultantes de quedas foi $28,1 \%$. É provável que características quanto ao local do estudo e tipo de serviço possam interferir nos resultados.

Os acidentes de transporte constituíram o segundo grupo mais freqüente entre as vítimas de causas externas atendidas nas unidades estudadas. O número de vítimas diárias de acidentes de transporte atendidas nas unidades de urgência e emergência em Cuiabá (treze) mostrou-se superior aos resultados dos estudos de Deslandes e Silva $^{8}(11,8 \%)$ e Mesquita Filho e Mello Jorge $(2007)^{22}$, porém inferior aos revelados para a Grande Cuiabá ${ }^{26}$ e Alta Floresta ${ }^{25}$, onde os acidentes de transporte foram responsáveis por $26,8 \%$ e $41,0 \%$ das causas externas, respectivamente. Esses diferentes resultados evidenciam a necessidade de se considerar políticas de prevenção e controle de acidentes de trânsito também diferenciados, já que os resultados parecem ser influenciados pelas diferenças quanto às características da malha viária, da estrutura da população, e mesmo dos serviços de saúde. 
Certamente, o número de vítimas de acidentes de transporte é ainda maior, haja vista que muitas mortes ocorrem no próprio local do evento, não sendo, portanto, encaminhadas às unidades de urgência $\mathrm{e}$ emergência.

Os motociclistas e ciclistas foram as principais vítimas de acidentes de transporte; resultados semelhantes foram observados em outros estudos ${ }^{25-27}$.

Referente ao grande número de atendimentos de motociclistas, alguns autores ${ }^{27}$ afirmam que esse fato se deve à dificuldade de visualização da motocicleta, maior exposição corpórea dos motociclistas e maior prevalência de comportamento inadequado desses no trânsito. Os acidentes envolvendo motocicletas são um problema emergente decorrente do crescimento do uso deste veículo não somente para lazer, mas principalmente para o trabalho nas atividades de serviços de "motoboy" e "mototaxi" ${ }^{27}$. $\mathrm{Na}$ Grande Cuiabá, as motocicletas representavam cerca de $11,0 \%$ da frota total de veículos em 2000 e, em 2005, esse percentual elevase para $21,3 \%^{26}$. Neste mesmo ano, estudo apresentado pela Associação Brasileira de Medicina de Tráfego revela que Cuiabá tem a segunda maior frota de motocicletas entre as capitais brasileiras ${ }^{16}$.

Os acidentes de transporte se configuram como a segunda causa de mortalidade por causa externa no país, e se apresenta com tendência crescente em Cuiabá ${ }^{5,11,14}$. Eles também foram o segundo grupo de internações por causas externas em Cuiabá, no ano de $2007^{14}$.

Por outro lado, as agressões, que foram pouco representativas no atendimento de urgência e emergência, revelado nesse estudo, têm se configurado, nas últimas décadas, como a principal causa de mortalidade, entre os tipos de causas externas no Brasil ${ }^{5,7,9,14}$ e em Cuiabá ${ }^{14}$. Percentuais semelhantes aos apresentados neste estudo foram encontrados por outros autores ${ }^{22,23,26}$. Contudo, percentuais baixos de atendimentos de agressão em unidades de urgência e emergência podem estar relacionados à subnotificação; seja por parte das vítimas, por temerem retaliação por parte do agressor, ou dos profissionais de saúde, que também podem ser intimidados pelo agressor, que algumas vezes acompanha a própria vítima ao serviço de saúde ${ }^{22}$.

Os resultados deste e de outros estu$\operatorname{dos}^{25,26}$ quanto à utilização de drogas em vítimas de agressão mostram que estratégias de redução da violência devem fundamentalmente abordar alguma forma de intervenção sobre o consumo de álcool/droga.

Entre as vítimas atendidas nas unidades de urgência e emergência, há predominância do sexo masculino (2:1); em outros estudos também foi identificado o predomínio do sexo masculino ${ }^{8,22,25-27}$. Este comportamento quanto ao gênero é diferente apenas para os casos de lesões autoprovocadas, nos quais há predominância do sexo feminino. Resultados semelhantes são apresentados por outros estudos que enfocam lesões autoprovocadas não fatais ${ }^{2,25}$. Problemas de relacionamento familiar ou de namoro, entre os jovens, e problemas de ordem financeira e/ou conjugais entre os adultos são prováveis fatores que levam à tentativa de suicídio ${ }^{22}$.

A preponderância do sexo masculino também é verificada nos dados de morbidade hospitalar e mortalidade por causas externas em Cuiabá ${ }^{14}$. No entanto, a razão entre os sexos nos atendimentos em unidades de urgência e emergência é menor que na morbidade hospitalar (3) e na mortalidade $(5,7)$, evidenciando a importância de se analisar diversas fontes de dados.

Os mais jovens, incluindo crianças (menores de 10 anos) e adolescentes (10 a 19 anos), são as principais vítimas atendidas nas unidades de urgência e emergência; resultado que se diferencia da morbidade hospitalar e mortalidade em Cuiabá, onde predomina o grupo de 20 a 39 anos $^{20}$. Outros autores $^{22,26}$ também mostram uma maior prevalência em jovens, se assemelhando aos resultados deste estudo. Mesquita e Mello Jorge $(2007)^{22}$ revelam que a idade média de vítimas de causas de externas atendidas em uma unidade de pronto atendimento foi de 26,3 anos, com desvio padrão de 19,4 anos, o 
que mostra uma dispersão importante entre as idades. Vários são os motivos apontados para a alta prevalência de acidentes e violência entre a população de menos idade, seja pelo abuso ou descuido infantil, caracterizada por agressão física, sexual, verbal, emocional e abandono, seja por características individuais, experiências familiares, acesso a armas, álcool e drogas ${ }^{22,28}$.

$\mathrm{O}$ fato de a maioria dos acidentes e violências ter ocorrido em residência, local onde, em geral, as pessoas deveriam se sentir em maior segurança, traz uma reflexão no sentido de prevenção e controle desses eventos, que não se pode restringir às áreas coletivas.

Nesse estudo, mesmo tendo sido observado que os fins de semana contribuíram com quase a metade dos atendimentos, deve ser destacado que, nas sextas-feiras, foi encontrado o menor percentual entre os dias da semana $(12,0 \%)$, diferenciando-se assim de alguns resultados da literatura ${ }^{8,27}$.

A quase totalidade das vítimas recebeu alta após o atendimento nas unidades de urgência e emergência, possivelmente devido ao caráter leve das lesões. Portanto, muitos desses atendimentos poderiam ser realizados em Unidades Básicas de Saúde ou Policlínicas, e não no Pronto Socorro, como foi o observado. Esse fato aponta para a necessidade de reorganização dos serviços com vistas à maior resolubilidade em todos os níveis.

Estudando acidentes de transporte, Andrade e Mello Jorge (2001) ${ }^{27}$ e Deslandes e Silva $(2000)^{8}$ encontraram letalidade de $1,8 \%$ e 3,4\% respectivamente. Esses resultados mostram-se muito distintos dos apresentados neste estudo, onde a letalidade tanto por causas externas em geral quanto para acidentes de transporte foi de $0,4 \%$; este fato sugere diferenças no nível de complexidade entre os serviços estudados. Outros aspectos podem ter influenciado esse resultado, entre eles, o direcionamento, pelo SAMU ou SIATE, de vítimas para hospitais privados ou ainda diretamente para internação, caso que ocorre com certa freqüência, via Central de Regulação; além disso, se houve óbito no local, devido à gravidade do caso, essas vítimas podem ter sido encaminhadas diretamente ao Instituto Médico Legal.

\section{Considerações Finais}

Os acidentes e a violência configuram problema de saúde pública de grande magnitude e transcendência, com forte impacto na morbidade e na mortalidade da população brasileira. Diminuir este impacto é um dos principais desafios.

Freqüentemente, as ações de prevenção e controle de acidentes e violência são priorizadas, considerando-se as mortes decorrentes desses eventos, tendo em vista a maior disponibilidade e qualidade dos dados de mortalidade. Por outro lado, quando se analisam os dados tendo como foco as vítimas não fatais, o quadro epidemiológico se apresenta muito diferente, como demonstrado nesse estudo, e, portanto, precisa ser considerado quando da definição de prioridades.

No Brasil, são raros os locais que coletam dados sistemáticos e rotineiros sobre causas externas e vítimas atendidas em unidades de urgência e emergência; contudo, o conhecimento do perfil dessa população e desses eventos contribui para o monitoramento dos acidentes e violências, tornando assim uma importante fonte de informação. Neste sentido, este estudo fomenta uma reflexão sobre a relevância da implantação de um sistema de informação para o conhecimento de dados provenientes da demanda das unidades de urgência e emergência, visando a caracterização dessa demanda e possibilitando a complementação dos dados de mortalidade e morbidade hospitalar existentes, contribuindo para a prevenção e controle de acidentes e violências. 


\section{Referências}

1. [OPS] Organización Panamericana de la Salud. Programa Especial de Análises de Salud. Situación de salud en las Américas: indicadores básicos 1999. Washington, DC; 1999.

2. [OMS] Organização Mundial da Saúde. Relatório mundial sobre violência e saúde. Genebra; 2002.

3. Mello Jorge MHP, Cascão AM, Silva RC. Acidentes e violências: um guia para o aprimoramento da qualidade de sua informação. São Paulo: MS/USP/OPS/OMS; 2003. Série divulgação v 10.

4. Souza ER, Minayo MCS, Silva CMFP, Reis AC, Malaquias JV, Veiga JPC et al. Análise temporal da mortalidade por causas externas no Brasil: décadas de 80 e 90. In: Minayo MCS, Souza ER, organizadores. Violência sob o olhar da saúde: a infrapolítica da contemporaneidade brasileira. Rio de Janeiro: Editora FIOCRUZ; 2003. p 83-107.

5. [MS] Ministério da Saúde. Secretaria de Vigilância em Saúde. Saúde Brasil 2005: uma análise da situação de saúde. Brasília, DF; 2005.

6. Mello Jorge MHP, Koizumi MS. Acidentes de transito no Brasil: breve análise de suas fontes de dados. Rev ABRAMET. 2002; 38:53-62.

7. Souza ER, Ximenes LF, Alves F, Magalhães C, Bilate D, Szuchmacher AM, Malaquias J. Avanços do conhecimento sobre as causas externas no Brasil e no mundo: enfoque quantitativo e qualitativo. In: Minayo MCS, Souza ER, organizadores. Violência sob o olhar da Saúde: A infrapolítica da contemporaneidade brasileira. Rio de Janeiro: Editora FIOCRUZ; 2003. p. 131-60.

8. Deslandes SF, Silva CMFP. Análise da morbidade hospitalar por acidentes de trânsito em hospitais públicos do Rio de Janeiro, RJ, Brasil. Rev Saúde Pública. 2000; 34: 367-72.

9. Mello Jorge MHP, Gotlieb SL. As condições de saúde no Brasil: retrospecto de 1975 a 1995. Rio de Janeiro: Editora FIOCRUZ; 2000.

10. Ministério da Saúde. Secretaria de Vigilância em Saúde. Análise da morte violenta segundo raça/cor: acidentes por transporte terrestre e análise da tendência da morte violenta: acidentes por transporte terrestre. In: Saúde Brasil, 2005. Brasília: Ministério da Saúde; 2005.

11. Souza ER, Minayo MCS, Malaquias JV. Violência no trânsito: expressão da violência social. In: [MS] Ministério da Saúde. Secretaria de Vigilância em Saúde. Impacto da violência na saúde dos brasileiros. Brasília, DF; 2005. p. 279-312.

12. [MS] Ministério da Saúde. Mortalidade por armas de fogo no Brasil: 1991-2000. Brasília, DF; 2004.
13. Souza ER, Lima MLC, Costa da Veiga JP. Violência interpessoal: homicídios e agressões. In: [MS] Ministério da Saúde. Secretaria de Vigilância em Saúde. Impacto da violência na saúde dos brasileiros. Brasília, DF; 2005. p. 171-203.

14. [MS] Ministério da Saúde. DATASUS [homepage na Internet]. Brasil; 2008. [acesso em 10 abr 2008]. Disponível em: http://w3.datasus.gov.br/datasus.

15. Waiselfisz JJ. Mapa da Violência dos Municípios Brasileiros. [OEI] Organização dos Estados IberoAmericanos para a Educação, a Ciência e a Cultura. Brasília, DF; 2007.

16. Mello Jorge MHP, Koizumi MS. Acidentes de Trânsito no Brasil: um Atlas de sua distribuição. [ABRAMET] Associação Brasileira de Medicina de Tráfego. São Paulo, 2007.

17. Ministério da Saúde. Secretaria de Vigilância em Saúde. Departamento de Análise de Situação em Saúde. Brasília: Projeto Vigilância de acidentes e violências rede serviço sentinela de acidentes e violências. Brasília, 2006.

18. [OMS] Organização Mundial da Saúde. Centro Colaborador da OMS para a Classificação de Doenças em Português. Classificação estatística internacional de doenças e problemas relacionados à Saúde: $10^{\mathrm{a}}$ revisão, 1995. São Paulo, SP; 1995.

19. Souza ER, Minayo MCS, Njaine K, Cruz AJM, Silva HB, Santos FCL, Moreira CO. Tendências da produção científica brasileira sobre violência e acidentes na década de 90. In: Minayo MCS e Souza ER, organizadores. Violência sob o olhar da saúde: a infrapolítica da contemporaneidade brasileira. Rio de Janeiro: Editora FIOCRUZ; 2003. p. 49-81.

20. Mello Jorge MHP, Koizumi MS,. Gastos governamentais do SUS com internações hospitalares por causas externas: análise no Estado de São Paulo, 2000. Rev Bras Epidemiol. 2004; 7:228-38.

21. Minayo MCS, Souza ER, Malaquias JV, Reis AC, Santos NC, Veiga JPC et al. Análise da morbidade hospitalar por lesões e envenenamentos no Brasil em 2000. In: Minayo MCS, Souza ER, organizadores. Violência sob o olhar da saúde: a infrapolítica da contemporaneidade brasileira. Rio de Janeiro: Editora FIOCRUZ; 2003. p 109-22.

22. Mesquita Filho M, Mello Jorge MHP. Características da morbidade por causas externas em serviço de urgência. Rev Bras Epidemiol. 2007; 10:579-91.

23. Mello Jorge MHP, Gawryszewski VP, Koizume MS. Contribuição do setor saúde no atendimento às vítimas e prevenção da violência: relatório apresentado a FAPESP. São Paulo; 2006.

24. Melione LPR. Morbidade hospitalar por causas externas no Sistema Único de Saúde em São José dos Campos [dissertação de mestrado]. São Paulo: Faculdade de Saúde Pública da Universidade de São Paulo; 2006. 
25. Marchese VS. A morbidade e a mortalidade por acidentes e violências no município de Alta Floresta - MT [dissertação de mestrado]. Cuiabá: Instituto de Saúde Coletiva, Universidade Federal de Mato Grosso; 2007.

26. Soares BAC. Morbidade e mortalidade por acidentes e violências na Grande Cuiabá-MT [dissertação de mestrado]. Cuiabá: Instituto de Saúde Coletiva, Universidade Federal de Mato Grosso; 2007.
27. Andrade SM, Mello Jorge MHP. Acidentes de transporte terrestre em município da Região Sul do Brasil. Rev Saúde Pública. 2001;35:318-20.

28. Assis SG, Deslandes SF, Santos NC. Violência e adolescência: sementes e frutos de uma sociedade desigual. In: [MS] Ministério da Saúde. Secretaria de Vigilância em Saúde. Impacto da violência na saúde dos brasileiros. Brasília, DF; 2005. p. 79-105.

Recebido em: 24/11/07

Versão final reapresentada em: 23/05/08

Aprovado em: 25/06/08 